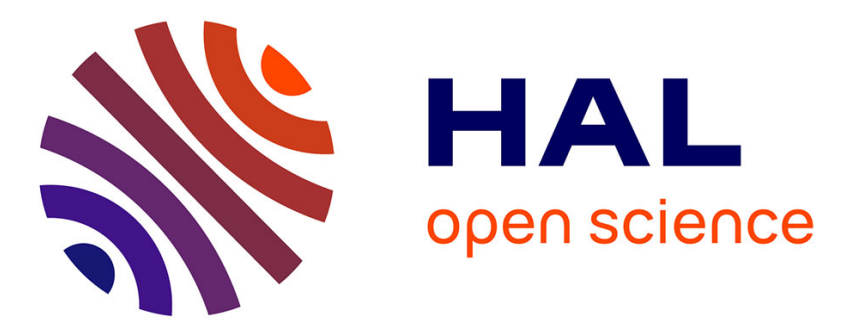

\title{
Caching Games between Content Providers and Internet Service Providers
}

\author{
Vaggelis G Douros, Salah Eddine Elayoubi, Eitan Altman, Yezekael Hayel
}

\section{To cite this version:}

Vaggelis G Douros, Salah Eddine Elayoubi, Eitan Altman, Yezekael Hayel. Caching Games between Content Providers and Internet Service Providers. Valuetools, Oct 2016, Taormina, Italy. hal01404872

\section{HAL Id: hal-01404872 \\ https://hal.inria.fr/hal-01404872}

Submitted on 29 Nov 2016

HAL is a multi-disciplinary open access archive for the deposit and dissemination of scientific research documents, whether they are published or not. The documents may come from teaching and research institutions in France or abroad, or from public or private research centers.
L'archive ouverte pluridisciplinaire $\mathbf{H A L}$, est destinée au dépôt et à la diffusion de documents scientifiques de niveau recherche, publiés ou non, émanant des établissements d'enseignement et de recherche français ou étrangers, des laboratoires publics ou privés. 


\title{
Caching Games between Content Providers and Internet Service Providers
}

\author{
Vaggelis G. Douros ${ }^{1}$, Salah Eddine Elayoubi ${ }^{1}$, Eitan Altman², Yezekael Hayel ${ }^{3}$ \\ ${ }_{1}^{1}$ Orange Labs, Châtillon, France; \{evangelos.douros, salaheddine.elayoubi\}@orange.com \\ ${ }^{2}$ INRIA, Sophia Antipolis, France; eitan.altman@sophia.inria.fr \\ ${ }^{3}$ University of Avignon, Avignon, France; yezekael.hayel@univ-avignon.fr
}

\begin{abstract}
We consider a scenario where an Internet Service Provider (ISP) serves users that choose digital content among $M$ Content Providers (CP). In the status quo, these users pay both access fees to the ISP and content fees to each chosen CP; however, neither the ISP nor the CPs share their profit. We revisit this model by introducing a different business model where the ISP and the CP may have motivation to collaborate in the framework of caching. The key idea is that the ISP deploys a cache for a CP provided that they share both the deployment cost and the additional profit that arises due to caching. Under the prism of coalitional games, our contributions include the application of the Shapley value for a fair splitting of the profit, the stability analysis of the coalition and the derivation of closed-form formulas for the optimal caching policy.

Our model captures not only the case of non-overlapping contents among the $\mathrm{CPs}$, but also the more challenging case of overlapping contents; for the latter case, a non-cooperative game among the CPs is introduced and analyzed to capture the negative externality on the demand of a particular $\mathrm{CP}$ when caches for other CPs are deployed.
\end{abstract}

\section{Keywords}

Coalitional game theory, Shapley value, Nash Equilibrium

\section{INTRODUCTION AND CONTRIBUTIONS}

The Internet ecosystem is classically described as an interconnection of Autonomous Systems (ASs) [6], exchanging traffic through peering (free of charge) contracts or customerprovider contracts. The ASs have been classified by [1] into five categories: Large Transit Providers, Small Transit Providers, Access/Hosting providers, Enterprise Customers, and Content Providers. From an AS perspective, the ASs that are involved in a customer-provider link with a transit Internet Service Provider (ISP) pay this latter for the traffic volume that flows through this link.
From an end user perspective, the current Internet pricing model involves two customer-provider relationships: i) Access fees, where end users pay a fee to access ISPs for the connectivity services, based, in general, on flat offers. ii) Content pricing, where end users pay (directly or indirectly via advertisement) to the Content Providers (CP).

An alternative Internet economic model has been proposed by $\mathrm{Ma}$ et al. in [4]; the idea being that end users pay for end-to-end services provided by a set of ISPs, and ISPs collectively share the revenue generated from these customers based on a profit distribution mechanism. Notions from the coalitional game theory [8] have been used for designing the profit distribution mechanism to ensure fairness and efficiency properties.

Towards this direction, the goal of our work is to analyze a business model where the ISP and the CPs collaborate by sharing the cache cost/profit deployment. We use coalitional game theory to model the interactions between them and we make the following contributions:

For the case that there is a unique CP: i) We use the Shapley value [8] to propose a fair splitting of the-due to caching-profit between the CP and the ISP. ii) We compute the Nash Bargaining Solution [8] that has also appealing properties, showing that it coincides with the Shapley value for our model. iii) We analyze the stability of the Shapley value, showing under which conditions it belongs to the core [8] of the game. iv) We compute the optimal caching policy that maximizes the revenue of both the ISP and the CP. Our simulations show that there is a significant increase in the profit of the ISP and the $\mathrm{CP}$ with respect to the case that there is no cache deployment.

For the case that there are multiple CPs: i) We compute the Shapley value. ii) We analyze the non-cooperative game [3] that arises due to the competition among the CPs. iii) We prove that this game admits always a Nash Equilibrium (NE). iv) We derive a necessary and sufficient condition for the uniqueness of the NE. v) We propose a best-response dynamics scheme that converges fast to the NE.

\section{BASELINE MODEL: THE CASE OF ONE CONTENT PROVIDER}

\subsection{Preliminaries}

We consider a scenario with one ISP that serves $J$ users and one CP that offers additional content (movies, sports, etc.) to these users (see Figure 1); let $N$ be the number of the items that the CP sells and let $P$ be the price per item. Each user $j$ pays both access fees to the ISP and content 


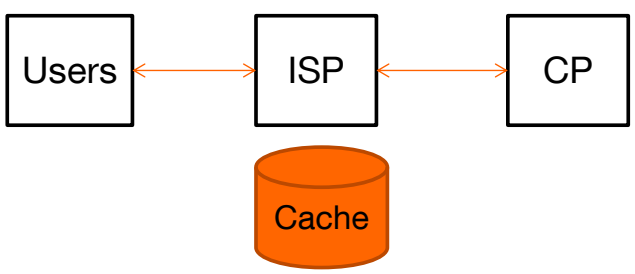

Figure 1: Baseline scenario: the case of $1 \mathrm{CP}$.

fees to the CP. Note that we do not take into consideration indirect costs (e.g., from ads shown as part of search results).

Then, we compute the profit for the ISP and the CP, expressed as a utility function that captures the difference between the income minus the expenses. The utility for the $\mathrm{CP}$, denoted by $U_{\mathrm{CP}}$, is the difference between the content fees (i.e., the total demand $D$ for the $N$ items from the $J$ users multiplied by the price per item $P$ ) that the users of the ISP pay to him $^{1}$ minus his operational expenses, denoted by $O$. Therefore, we get that: $U_{\mathrm{CP}}=D P-O$.

On the other hand, the utility of the ISP, denoted by $U_{\text {ISP }}$, is the difference between the sum of the access fee $\pi_{j}$ that each user $j$ pays to the ISP minus the product of the backhaul bandwidth $B$ for serving the demand $D$ for the contents of the CP multiplied by the unit backhaul bandwidth cost $b$. Therefore, we get that: $U_{\mathrm{ISP}}=\sum_{j=1}^{J} \pi_{j}-B b$.

\subsection{The Impact of Caching}

In this section, we examine the impact of caching on the utility functions of the ISP and the CP. In our context, the adoption of caching incurs three changes in the model:

- There is a cache deployment cost; for a cache of size $C$ and with a unit cache cost $s$, this is equal to $s C$.

- The total demand $D$ for the $N$ items of the CP will change; this is due to the fact that the demand is based on the perceiving Quality-of-Experience (QoE) of the users and when a cache for the $\mathrm{CP}$ is deployed by the ISP, this has a direct impact on the users' QoE.

- The backhaul bandwidth $B$ needed will change due to caching, since some of the requests for the contents of the $\mathrm{CP}$ could be served directly by the cache.

So as to quantify the above factors, we examine the case that the ISP deploys a cache of size $C$, storing proactively the $C$ most popular items out of the $N$ items of the CP. Let $D^{C}$ be the new demand for the contents of the CP; the superscript $C$ is an abbreviation for "caching". The quantity $D^{C}$ equals to: $D^{C}=(1+\Delta) D$. The parameter $\Delta$ corresponds to a QoE factor that reflects the change on the demand for the contents of the CP.

We choose the hit ratio as the QoE metric, which is a standard metric for the efficiency of the cache. The hit ratio $h$ is defined as the fraction of the number of requests for the $N$ items that have been found in the cache over the total number of requests for the $N$ items; clearly, $h$ is a number between 0 and 1 ; the larger the hit ratio, the happier are the users; chances are that they get the content that they want directly from the cache, without need to wait for the ISP to communicate with the $\mathrm{CP}$ to send the requested content

\footnotetext{
${ }^{1}$ We use the pronoun "he" when we refer to the ISP/CP,
} implying the owner of the ISP/CP. back to the ISP. Therefore, this will have a positive impact on the demand for the contents of the CP.

Based on the above discussion, we define the parameter $\Delta$ to be proportional to the hit rate $h$ as: $\Delta=F h$, with $F$ being a non-negative constant. Clearly, the lower bound of $\Delta$ is zero; in this case, the total demand for the contents of the CP $D^{C}$ with caching is the same with the total demand $D$ without caching.

We then compute the formula for the new backhaul bandwidth, denoted by $B^{C}$; it corresponds to the number of cases where the request for an item of the $\mathrm{CP}$ has not been served by the cache; these cases correspond to the miss ratio of the cache of $\mathrm{CP}$, defined as: $1-h$. Therefore, $B^{C}$ is computed by the formula:

$$
B^{C}=B(1-h) .
$$

\subsection{Update on the Utility Functions}

Using the equations of the previous section (a summary of the notation used so far is provided in Table 1), we can rewrite the utility functions of the $\mathrm{CP}$ and the ISP.

$$
\begin{array}{r}
U_{\mathrm{CP}}^{C}=(1+\Delta) D P-O, \\
U_{\mathrm{ISP}}^{C}=\sum_{j=1}^{J} \pi_{j}-s C-B(1-h) b .
\end{array}
$$

By computing the difference of the utilities with caching versus without caching, we get:

$$
\begin{array}{r}
U_{\mathrm{CP}}^{C}-U_{\mathrm{CP}}=\Delta D P, \\
U_{\mathrm{ISP}}^{C}-U_{\mathrm{ISP}}=-s C+h B b .
\end{array}
$$

It is interesting that the deployment of the cache incurs always a positive externality for the income of $\mathrm{CP}$, since his profit will increase (in the worst case, it will remain stable). On the other hand, the deployment of the cache is beneficial for the ISP if and only if the backhaul bandwidth savings $h B b$ are larger than the cache cost deployment $s C$.

\subsection{A Different Business Model: Sharing Cache Cost/Profit between the ISP and the CP}

\subsubsection{Preliminaries}

In this section, we propose a different business model where the CP and the ISP are willing to split the cost/profit that arises due to caching. Therefore, they are willing to form a coalition, sharing the quantity $\Phi$, which is equal to:

$$
\Phi=U_{\mathrm{CP}}^{C}-U_{\mathrm{CP}}+U_{\mathrm{ISP}}^{C}-U_{\mathrm{ISP}}=\Delta D P-s C+h B b .
$$

The motivation for the adoption of the model is to ensure the alignment of the interest of both the ISP and the CP to deploy a cache. In the status "quo" model where the ISP pays exclusively for the cache deployment, we have shown in the previous section that when the cache cost is larger than the backhaul bandwidth savings, the ISP does not have motivation to deploy the cache. The question is whether the participation of the $\mathrm{CP}$ into the cache cost deployment will broaden the number of cases where the ISP is willing to deploy the cache.

Note that the quantity $\Phi$ that corresponds to the additional cost/profit due to caching for both the ISP and the $\mathrm{CP}$ will be the same either when the ISP pays for the cache deployment or when the ISP and the CP share the cache cost/profit (or even when the $\mathrm{CP}$ offers the cache for free 
Table 1: Summary of the Notation.

\begin{tabular}{|c|c|c|}
\hline Symbol & Domain & Description \\
\hline \multicolumn{3}{|c|}{ Case 1: No cache is deployed in the ISP network } \\
\hline 1 & \multirow{6}{*}{$\begin{array}{l}\text { Positive integer } \\
\quad \geq 0 \\
>0 \\
>0\end{array}$} & One Content Provider $(\mathrm{CP})$ \\
\hline$N$ & & Number of items of the CP \\
\hline$D$ & & Total demand for the $N$ items of the $\mathrm{CP}$ \\
\hline$P$ & & Price for each item of the CP \\
\hline$O$ & & Fixed expenses of the $\mathrm{CP}$ \\
\hline 1 & & One Internet Service Provider (ISP) \\
\hline$J$ & \multirow{2}{*}{ Positive integer } & Number of users that the ISP serves \\
\hline$\pi_{j}$ & & Price that each user $j$ pays to the ISP \\
\hline$B$ & $\geq 0$ & Backhaul bandwidth needed to serve the demand $D$ \\
\hline$b$ & $>0$ & Unit backhaul bandwidth cost \\
\hline \multicolumn{3}{|c|}{ Case 2: Cache is deployed in the ISP network } \\
\hline \multirow{8}{*}{$\begin{array}{c}s \\
C \\
h=\left(\frac{C}{N}\right)^{1-a} \\
a \\
D^{C}=(1+\Delta) D \\
B^{C}=B(1-h) \\
\Delta=F h \\
F\end{array}$} & $>0$ & Unit cache cost \\
\hline & $\in[0, N]$ & Cache size of the CP in the ISP network \\
\hline & $\in[0,1]$ & Hit rate for the $\mathrm{CP}$ \\
\hline & $\in(0,1)$ & Zipf parameter \\
\hline & $\geq 0$ & New demand for the $N$ items of the CP \\
\hline & $\geq 0$ & New backhaul bandwidth needed to serve the demand $D^{C}$ \\
\hline & $\geq 0$ & Reflects the changes on the demand \\
\hline & $\geq 0$ & Constant multiplier \\
\hline
\end{tabular}

to the ISP network-this is the case with Google nowadays). The difference through our model lies on how to split this additional cost/profit.

\subsubsection{Shapley Value}

To decide how $\Phi$ should be split between the CP and the ISP, we use a well known solution concept from the coalitional game theory, the Shapley value formula. This distribution scheme, defined by L. Shapley [8], is an interesting solution in coalition games thanks to its fairness. It is the unique distribution satisfying the following axioms: efficiency (i.e., the total surplus is allocated), symmetry (i.e., players having the same contribution in the coalition are paid equally), dummy (i.e., players that do not have a contribution in the considered coalition are not paid), and additivity (i.e., if a game is composed of two sub-games, players are paid the sum of their shares in both sub-games). Therefore, each player receives a profit share proportional to its contribution in the network setting and the added value it brings to the overall value chain.

In the following Theorem, we compute the Shapley value.

THEOREM 1. Let $\Phi_{\mathrm{CP}}$ be the quantity that the CP receives and $\Phi_{\mathrm{ISP}}$ be the quantity that the ISP receives after the application of the Shapley value on the quantity $\Phi$. Then:

$$
\begin{aligned}
\Phi_{\mathrm{CP}} & =\frac{1}{2}(\Delta D P-s C+h B b), \\
\Phi_{\mathrm{ISP}} & =\frac{1}{2}(\Delta D P-s C+h B b)
\end{aligned}
$$

Proof. The Shapley value for a player $i$ is defined as:

$$
\Phi_{i}=\frac{1}{N !} \sum_{R}\left[u\left(P_{i}^{R} \cup(i)\right)-u\left(P_{i}^{R}\right)\right]
$$

where $N$ ! is the number of orders $R$ of the players, $P_{i}^{R}$ is the set of players in $N$ that precede $i$ in the order $R$, and the function $u(\cdot)$ corresponds to the utility function of the
Table 2: Shapley Value for the CP.

\begin{tabular}{|c||c|}
\hline Orders & Marginal Contribution of the CP \\
\hline $\mathrm{CP}$, ISP & 0 \\
\hline ISP, $\mathrm{CP}$ & $\Delta D P-s C+h B b$ \\
\hline
\end{tabular}

coalition. In our case, there are two players (the CP and the ISP) and $N=2$ orders per player. In Table 2, we compute the marginal contribution $u\left(P_{i}^{R} \cup(i)\right)-u\left(P_{i}^{R}\right)$ for each possible order for the CP. By definition, the utility function of the empty set is zero. When the CP is first, his marginal contribution is zero, since the coalition has not been formed yet. When the CP is second, the coalition has been formed and the marginal contribution of the $\mathrm{CP}$ is equal to the quantity $\Phi$. Therefore:

$$
\Phi_{\mathrm{CP}}=\frac{1}{2}(0+\Phi)=\frac{1}{2}(\Delta D P-s C+h B b) .
$$

We can compute the Shapley value of the ISP similarly. Alternatively, we can exploit the efficiency axiom, i.e., using the fact that the Shapley value of the ISP is the difference between the total utility minus the Shapley value of the CP.

Therefore, the utility functions after the application of the Shapley value are:

$$
\begin{array}{r}
U_{\mathrm{CP}}^{C}=D P-O+\Phi_{\mathrm{CP}}=D P-O+\frac{1}{2}(\Delta D P-s C+h B b), \\
U_{\mathrm{ISP}}^{C}=\sum_{j=1}^{J} \pi_{j}-B b+\Phi_{\mathrm{ISP}}=\sum_{j=1}^{J} \pi_{j}-B b+ \\
\frac{1}{2}(\Delta D P-s C+h B b) .
\end{array}
$$

\subsubsection{Nash Bargaining Solution}

In this section, we present the outcome of splitting the cache cost/profit quantity $\Phi$ by using a different cooperative solution concept, the Nash Bargaining solution [8]. According to this, there are two players that demand a portion of 
the same good; if the total amount requested by the two players is less than what is available, then both players get their request; if not, neither player gets their request and there is a fixed disagreement outcome $d$ which will be the result.

Nash proposed that a solution to this problem should satisfy a number of axioms (invariant to affine transformations, Pareto optimality, independence of irrelevant strategies, and symmetry) and proved that the only formula that satisfies these axioms is

$$
\left(\Phi_{\mathrm{CP}}-d_{\mathrm{CP}}\right)\left(\Phi_{\mathrm{ISP}}-d_{\mathrm{ISP}}\right)
$$

with $\Phi_{\mathrm{CP}}, \Phi_{\mathrm{ISP}}$ being the portions of the quantity $\Phi$ that the $\mathrm{CP}$ and the ISP receives if the bargaining is successful and $d_{\mathrm{CP}}, d_{\mathrm{ISP}}$ being the disagreement points of the $\mathrm{CP}$ and the ISP.

In our case, the disagreement points correspond to the utility that the ISP and the CP get without caching. These are equal to:

$$
\begin{aligned}
& d_{\mathrm{CP}}=U_{\mathrm{CP}}^{C}-U_{\mathrm{CP}}=\Delta D P, \\
d_{\mathrm{ISP}}= & U_{\mathrm{ISP}}^{C}-U_{\mathrm{ISP}}=-s C+h B b .
\end{aligned}
$$

For these disagreement points, it is trivial to check that the Nash Bargaining Solution point coincides with the Shapley value (the proof is omitted due to space constraints).

\subsubsection{Shapley Value and the Core}

Though the Shapley value defines a fair way of dividing the utility between the (in our case two) members of the coalition, it ignores issues related to the stability. In other words, the question is whether the players are willing to form the coalition given the way that the Shapley value will divide their profits or some of them prefer to deviate and forming a different coalition or even they prefer to stay selfish. The players are willing to stay in a particular coalition if and only if the utility that they receive in this coalition is at least as large as the utility that they could earn by forming any other coalition (or by being selfish). If this is the case, then this splitting of the profit belongs to the core of the game [8]. In the following Theorem, we prove under which conditions the Shapley value belongs to the core of the game.

THEOREM 2. The Shapley value belongs to the core of the game if and only if the quantity $\Phi$ is non-negative.

Proof. By using the definition of the core of the game, we examine whether the utilities $U_{\mathrm{CP}}^{C}$ and $U_{\text {ISP }}^{C}$ are at least as large as in any other possible coalition. Since there are only two players, the only way to deviate from the coalition is to be selfish; Therefore, it should be:

$$
\begin{gathered}
U_{\mathrm{CP}}^{C} \geq U_{\mathrm{CP}} \Leftrightarrow \Phi_{\mathrm{CP}} \geq 0, \\
U_{\mathrm{ISP}}^{C} \geq U_{\mathrm{ISP}} \Leftrightarrow \Phi_{\mathrm{ISP}} \geq 0 .
\end{gathered}
$$

Since $\Phi_{\mathrm{CP}}=\Phi_{\mathrm{ISP}}=\frac{\Phi}{2}$, the theorem holds if and only if $\Phi$ is non-negative.

\subsubsection{On the Optimal Caching Policy}

In this section, we compute the optimal caching policy, i.e., the optimal choice of the cache size $C$ that maximizes the utility functions of the ISP and the CP. In order to provide a closed-form formula, we need to give an expression of the hit rate $h$ as a function of the cache size $C$. We use the approximation that is given in [2]; the authors assume that:

- the $N$ items of the CP are of equal size,

- their popularities follow a $\operatorname{Zipf}(a)$ distribution with parameter $a$ being a positive variable that belongs to $(0,1)$,

- the number of items $N$ is large

Then, they approximate the cache hit rate $h(C)$, denoted for simplicity as $h$, as:

$$
h=\left(\frac{C}{N}\right)^{1-a} .
$$

THEOREM 3. The caching size $C^{*}$ that maximizes the utility of both the ISP and the CP is:

$$
C^{\star}=\min \left\{N, \sqrt[a]{\frac{(1-a)(F D P+B b) N^{a}}{s}}\right\} .
$$

Proof. We rewrite $U_{\mathrm{CP}}^{C}$ and $U_{\mathrm{ISP}}^{C}$ by using the expression of the hit rate $h$.

$$
\begin{array}{r}
U_{\mathrm{CP}}^{C}=D P-O+\frac{1}{2}\left(F\left(\frac{C}{N}\right)^{1-a} D P-s C+\left(\frac{C}{N}\right)^{1-a} B b\right), \\
U_{\mathrm{ISP}}^{C}=\sum_{j=1}^{J} \pi_{j}-B b+\frac{1}{2}\left(F\left(\frac{C}{N}\right)^{1-a} D P-s C+\left(\frac{C}{N}\right)^{1-a} B b\right) .
\end{array}
$$

Then, we get the first derivative of the utilities (denoted by $U_{\mathrm{CP}}^{C^{\prime}}$ and $\left.U_{\text {ISP }}^{C^{\prime}}\right)$ with respect to $C$ and set them equal to zero.

$$
U_{\mathrm{CP}}^{C^{\prime}}=0 \Leftrightarrow C=\sqrt[a]{\frac{(1-a)(F D P+B b) N^{a}}{s}}=C^{\diamond} .
$$

Then, $U_{\mathrm{CP}}^{C^{\prime}}$ is positive in $\left(0, C^{\diamond}\right)$ and negative in $\left(C^{\diamond}, N\right)$. We distinguish two cases:

1. If $C^{\diamond}$ is larger than $N$, then the caching size $C^{\star}$ that maximizes $U_{\mathrm{CP}}^{C}$ is $N$.

2. If $C^{\diamond}$ is smaller than $N$, then $C^{\star}$ is equal to $C^{\diamond}$.

By combining the cases, we get that:

$$
C^{\star}=\min \left\{N, \sqrt[a]{\frac{(1-a)(F D P+B b) N^{a}}{s}}\right\} .
$$

For the case of the ISP, $U_{\text {ISP }}^{C^{\prime}}$ is equal to $U_{\mathrm{CP}}^{C^{\prime}}$ and we lead to the same caching size $C^{\star}$.

Note that the fact that the utilities are maximized for the same cache size implies that it is indifferent who controls the cache size (the CP or the ISP).

We did some simulations to measure the average profit increase per CP and ISP when the optimal caching policy $C^{*}$ is applied. We considered a system where the CP has a catalog of $N=100$ contents characterized by a zipf popularity law of parameter $a=0.8$. The ISP has $J=50$ users generating an initial demand of $D=1000$. Our results show an increase of the profits of the ISP and CP by $170 \%$ and $150 \%$, respectively, in comparison to the case where there is no caching. 


\section{MULTIPLE CONTENT PROVIDERS}

\subsection{Preliminaries}

We now examine the more general case (see Figure 2), where there is a unique ISP and $M$ CPs that offer additional content to the $J$ ISP users. This model is generalized as is for the case of multiple ISPs, provided that the users do not change ISP.

For the case that there is no caching, we redefine the utility functions using similar notation with the baseline scenario: For each CP $i$, the utility denoted by $U_{i}$ is equal to:

$$
U_{i}=D_{i} P_{i}-O_{i} .
$$

For the ISP, the only difference is that the total backhaul bandwidth $B$ is the sum of the backhaul bandwidth $B_{i}$ needed for the demand for contents of each CP $i$ :

$$
U_{\mathrm{ISP}}=\sum_{j=1}^{J} \pi_{j}-B b, B=\sum_{i=1}^{M} B_{i} .
$$

\subsection{The Impact of Caching}

We study scenarios where the ISP deploys a cache per CP; we do not consider the case that a cache is shared among different CPs, since this is not supported due to technical restrictions and security (cryptography) considerations. We distinguish two cases:

- When the contents of the CPs are non-overlapping (e.g., the first $\mathrm{CP}$ offers sports events, the second CP offers movies, the third CP offers music etc.), the baseline model is generalized as is; since the caches are independent, we should simply apply the baseline model for each cache to find out if the ISP and the CP $i$ have motivation to deploy the cache and, if so, how they should split the cache cost/profit and which is the optimal caching policy.

- When the contents of the CPs are overlapping. In this case, there is a coupling among the caches; this means that besides the coalitional game between each $\mathrm{CP} i$ and the ISP, there is a non-cooperative game among the CPs.

For the rest of the article, we deal only with the overlapping case. Firstly, we redefine the total demand after caching $D_{i}^{C}$ to be equal to:

$$
D_{i}^{C}=\left(1+\Delta_{i}\right) D_{i}
$$

As for the parameter $\Delta_{i}$, we assume, as previously, that it increases linearly with the cache hit rate $h_{i}$. Moreover, we assume that it decreases linearly with the sum of the hit rates of all other CPs; this is due to the fact that the caches of the other CPs create a negative externality to the demand of the CP $i$. Therefore, we get that:

$$
\begin{array}{r}
\Delta_{i}=F h_{i}-f \sum_{j \neq i} h_{j}, \\
F \geq(M-1) f \geq 0 \text { and } 0 \leq f \leq \frac{1}{M-1} .
\end{array}
$$

The global positive constants $F$ and $f$ are set to ensure that: i) Since the total demand $D_{i}^{C}$ cannot be negative, the parameter $\Delta_{i}$ cannot be lower than -1. ii) If all caches have the same hit rate, $D_{i}^{C}$ will be at least equal to $D_{i}$ (i.e, the

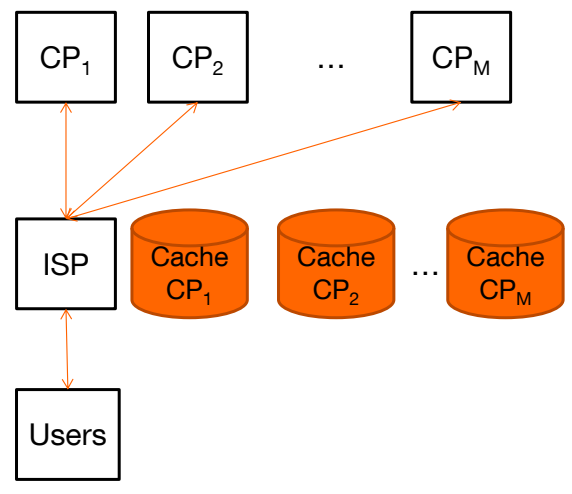

Figure 2: The case of multiple Content Providers.

demand with caching will be at least equal to the demand without caching).

We then define the new backhaul bandwidth needed $B_{i}^{C}$ :

$$
B_{i}^{C}=\left(1+\Theta_{i}\right) B_{i}\left(1-h_{i}\right) .
$$

The difference with the baseline scenario is the introduction of the term $1+\Theta_{i}$, with $\Theta_{i}$ being defined as:

$$
\Theta_{i}=-f \sum_{j \neq i} h_{j}
$$

The parameter $\Theta_{i}$ captures the fact that the backhaul bandwidth $B_{i}^{C}$ is reduced linearly with the sum of the hit rates of all other caches. Since $B_{i}^{C}$ is a non-negative quantity, the smaller possible value of $\Theta_{i}$ is -1 , which is ensured by the domain of the global constant $f$ (a summary of the additional notation is given in Table 3 ).

Therefore, the utility functions after caching are:

$$
\begin{array}{r}
U_{i}^{C}=D_{i}^{C} P_{i}-O_{i} . \\
U_{\text {ISP }}^{C}=\sum_{j=1}^{J} \pi_{j}-\sum_{i=1}^{M} s C_{i}-\sum_{i=1}^{M} B_{i}^{C} b .
\end{array}
$$

\subsection{A Different Business Model: Sharing Cache Cost/Profit between the ISP and the CP}

We extend the business model that we have presented for the baseline scenario with a unique CP; there is a coalitional game between the CP $i$ and the ISP with the view to sharing the cache cost/profit due to the deployment of a cache with the $C_{i}$ most popular items of the CP $i$ in the ISP network. Let $\Phi_{i}$ be the quantity that corresponds to this sharing, defined as:

$$
\begin{array}{r}
\Phi_{i}=U_{i}^{C}-U_{i}+U_{\text {ISP }}^{C}-U_{\text {ISP }} \\
=\Delta_{i} D_{i} P_{i}-s C_{i}-B_{i} b\left(-h_{i}-\Theta_{i} h_{i}+\Theta_{i}\right) .
\end{array}
$$

In the following Theorem, we compute the Shapley value.

THEOREM 4. Let $\Phi_{\mathrm{CP}}$ be the quantity that the CP $i$ receives and $\Phi_{\mathrm{ISP}}$ be the quantity that the ISP receives after the application of the Shapley value on $\Phi_{i}$. Then:

$$
\begin{array}{r}
\Phi_{\mathrm{CP}}=\frac{1}{2}\left[\left(\Delta_{i}+\Theta_{i}\right) D_{i} P_{i}-s C_{i}-B_{i} b\left(-h_{i}-\Theta_{i} h_{i}\right)\right] \\
\Phi_{\mathrm{ISP}}=\frac{1}{2}\left[\left(\Delta_{i}-\Theta_{i}\right) D_{i} P_{i}-s C_{i}-B_{i} b\left(-h_{i}-\Theta_{i} h_{i}+2 \Theta_{i}\right)\right]
\end{array}
$$

Proof. The main idea of the proof is similar with the proof of Theorem 1. In Table 4, we compute the marginal 
Table 3: Multiple CPs: Supplementary Notation.

\begin{tabular}{|c||c||c|}
\hline Symbol & Domain & Description \\
\hline$D_{i}^{C}=\left(1+\Delta_{i}\right) D_{i}$ & $\geq 0$ & New demand for the contents of the CP $i$ \\
$\Delta_{i}=F h_{i}-f \sum_{j \neq i} h_{j}$ & $\geq-1$ & Reflects the changes on the demand \\
$F$ & $\geq(M-1) f$ & Global constant multiplier \\
$f$ & $\in\left[0, \frac{1}{M-1}\right]$ & Global constant multiplier \\
$B_{i}^{C}=\left(1-\Theta_{i}\right) B_{i}\left(1-h_{i}\right)$ & $\geq 0$ & New bandwidth needed to serve the demand $D_{i}^{C}$ \\
$\Theta_{i}=-f \sum_{j \neq i} h_{j}$ & {$[-1,0]$} & Reflects the changes on the backhaul bandwidth \\
\hline
\end{tabular}

Table 4: Shapley Value for the CP $i$.

\begin{tabular}{|c||c|}
\hline Orders & Marginal Contribution of the CP $i$ \\
\hline CP $i$, ISP & $\Theta_{i} D_{i} P_{i}-0$ \\
\hline ISP, CP $i$ & $\Phi_{i}+B_{i} b \Theta_{i}$ \\
\hline
\end{tabular}

contribution of the CP $i$ for the two possible orders (the $\mathrm{CP}$ is first and the ISP is second, and vice versa). When the $\mathrm{CP} i$ joins first the coalition, his marginal contribution corresponds to the case that the ISP has not deployed a cache for his contents; however, the ISP may have deployed caches for the other CPs. In that case, $\Delta_{i}=\Theta_{i}$, since the hit rate $h_{i}$ is zero. Therefore, the marginal contribution is equal to $\Theta_{i} D_{i} P_{i}$ minus the utility of the empty set (which is zero by definition).

When the $\mathrm{CP} i$ joins second the coalition, his marginal contribution is equal to the quantity $\Phi_{i}$ minus the change of the utility of the ISP due to the change of the backhaul bandwidth, which is equal to the difference $B_{i}^{C}-B_{i}=\Theta_{i} B_{i}$.

Therefore, by using Table 4 and the definition of the Shapley value, we get that:

$$
\begin{array}{r}
\Phi_{\mathrm{CP}}=\frac{1}{2}\left(\Theta_{i} D_{i} P_{i}+\Phi_{i}+B_{i} b \Theta_{i}\right)= \\
\frac{1}{2}\left[\left(\Delta_{i}+\Theta_{i}\right) D_{i} P_{i}-s C_{i}-B_{i} b\left(-h_{i}-\Theta_{i} h_{i}\right)\right] .
\end{array}
$$

We can compute the Shapley value of the ISP similarly. Alternatively, we can exploit the efficiency axiom, stating that the Shapley value of the ISP is the difference between the total utility minus the Shapley value of the CP.

\subsection{A Non-Cooperative Game between the CPs}

\subsubsection{Preliminaries}

In the previous section, we have analyzed a coalitional game between the ISP and each CP $i$ to decide upon how they will distribute the cost/profit that arises due to caching. In this section, we model the interaction between the CPs as a non-cooperative game, defined formally as follows:

Definition 1: A normal form non-cooperative game G with a finite number of players consists of the following triplet: A set of players $M=\{1,2, \ldots, M\}$ and, for each player $i$, a set of strategies $S_{i}$, and a utility function $U_{i}(\cdot)$.

In our case: i) The players are the $M$ CPs. ii) The strategy of each player $i$ is the choice of the cache size $C_{i}$ that belongs to the closed interval $\left[0, N_{i}\right]$. iii) The utility function of each player $i$ is:

$$
U_{i}=D_{i} P_{i}-O_{i}+\frac{1}{2}\left(\Delta_{i}+\Theta_{i}\right) D_{i} P_{i}-s C_{i} .
$$

A powerful solution concept in non-cooperative game theory is the pure Nash Equilibrium (NE) [3] which predicts outcomes of games that are stable, in a sense described below.
Definition 2: The strategy vector $\mathbf{s}^{\star}=\left[s_{1}^{\star}, s_{2}^{\star}, \ldots, s_{M}^{\star}\right]$ is a pure NE for a game $G$ if and only if for each player $i$ and each other feasible strategy $s_{i}^{\diamond}$ of this player:

$$
\begin{array}{r}
U_{i}\left(s_{i}^{\star}, \mathbf{s}_{-\mathbf{i}}^{\star}\right) \geq U_{i}\left(s_{i}^{\diamond}, \mathbf{s}_{-\mathbf{i}}^{\star}\right), \text { where } \\
\mathbf{s}_{-\mathbf{i}}^{\star}=\left[s_{1}, s_{2}, \ldots, s_{i-1}, s_{i+1}, \ldots, s_{M}\right] .
\end{array}
$$

Consequently, a pure NE corresponds to a steady state of a game in the sense that no player has an incentive to change unilaterally his own strategy.

\subsubsection{Nash Equilibrium Existence}

In this section, we show that the non-cooperative game $G$ fulfils the properties of the Debreu-Glicksberg-Fan Theorem [3] and therefore admits a NE.

Theorem 5. The Game $G$ admits a NE.

Proof. We will use the following proposition, known as the Debreu-Glicksberg-Fan Theorem: Let $G$ be a normal form game, where, for each player $i$ : (i) The strategy set $S_{i}$ is compact and convex. ii) The utility function $U_{i}\left(s_{i}, s_{-i}\right)$ is continuous in $s_{-i}$. iii) The utility function $U_{i}\left(s_{i}, s_{-i}\right)$ is continuous and (quasi-)concave in $s_{i}$. Then, the game $G$ admits a pure NE.

We will show that these three properties hold in the game $G$. Firstly, the strategy set is the closed interval $\left[0, N_{i}\right]$, which is compact and convex.

Then, we rewrite the utility function $U_{i}$ :

$$
\begin{array}{r}
U_{i}=D_{i} P_{i}-O_{i}+\frac{1}{2}\left[\left(\Delta_{i}+\Theta_{i}\right) D_{i} P_{i}-s C_{i}\right. \\
\left.-B_{i} b\left(-h_{i}-\Theta_{i} h_{i}\right)\right]=D_{i} P_{i}-O_{i}+ \\
\frac{1}{2}\left[\left(F h_{i}-2 f \sum_{j \neq i} h_{j}\right) D_{i} P_{i}-s C_{i}-B_{i} b\left(-h_{i}+f \sum_{j \neq i} h_{j} h_{i}\right)\right] \\
=D_{i} P_{i}-O_{i}-f \sum_{j \neq i}\left(\frac{C_{j}}{N_{j}}\right)^{1-a} D_{i} P_{i}-\frac{1}{2} s C_{i}+ \\
\frac{1}{2}\left(F D_{i} P_{i}+B_{i} b-f \sum_{j \neq i}\left(\frac{C_{j}}{N_{j}}\right)^{1-a} B_{i} b\right) \frac{1}{N_{i}^{1-a}} C_{i}^{1-a} .
\end{array}
$$

Clearly, $U_{i}$ is continuous in $C_{i}$, as well as in $C_{-\mathbf{i}}$.

As for the third condition, since $U_{i}$ with respect to $C_{i}$ is twice differentiable, it is concave if and only if its second derivative $U_{i}^{\prime \prime}$ is non-positive. Indeed, we first compute the first derivative $U_{i}^{\prime}$ :

$$
\begin{array}{r}
U_{i}^{\prime}=-\frac{1}{2} s+\frac{1}{2}\left(F D_{i} P_{i}+B_{i} b-f \sum_{j \neq i}\left(\frac{C_{j}}{N_{j}}\right)^{1-a} B_{i} b\right) \\
\cdot \frac{1}{N_{i}^{1-a}}(1-a) C_{i}^{-a} .
\end{array}
$$


Then, we compute the second derivative $U_{i}^{\prime \prime}$ :

$$
\begin{array}{r}
U_{i}^{\prime \prime}=\frac{1}{2}\left(F D_{i} P_{i}+B_{i} b-f \sum_{j \neq i}\left(\frac{C_{j}}{N_{j}}\right)^{1-a} B_{i} b\right) \\
\cdot \frac{1}{N_{i}^{1-a}}(1-a)(-a) C_{i}^{-a-1} .
\end{array}
$$

Indeed, since

$$
f \sum_{j \neq i}\left(\frac{C_{j}}{N_{j}}\right)^{1-a} \leq \frac{1}{M-1}(M-1)=1,
$$

all product terms are non-negative, besides $-a$ which is negative, and therefore $U_{i}^{\prime \prime}$ is always non-positive.

\subsubsection{Nash Equilibrium Uniqueness}

In this section, we provide a necessary and sufficient condition for the uniqueness of the NE.

TheOREM 6. The Game $G$ admits a unique NE if and only if

$$
F \geq \frac{1-a}{a} \max _{i}\left\{\frac{N_{i} B_{i} b}{D_{i} P_{i}}\right\}
$$

Proof. We use the following proposition that holds for a concave game [3]: If a concave game satisfies the dominance solvability condition

$$
\frac{-\theta^{2} U_{i}}{\theta C_{i}^{2}} \geq \sum_{j \neq i}\left|\frac{\theta^{2} U_{i}}{\theta C_{j} \theta C_{i}}\right|
$$

then the game admits a unique NE.

In the previous theorem, we have computed $\frac{\theta^{2} U_{i}}{\theta C_{i}^{2}}$, so we need to compute $\frac{\theta^{2} U_{i}}{\theta C_{j} \theta C_{i}}$.

$$
\begin{gathered}
\frac{\theta^{2} U_{i}}{\theta C_{j} \theta C_{i}}=\frac{\theta}{\theta C_{j}}\left(-\frac{1}{2} s+\frac{1}{2}\left(F D_{i} P_{i}+B_{i} b-f \sum_{j \neq i}\left(\frac{C_{j}}{N_{j}}\right)^{1-a} B_{i} b\right)\right. \\
\left.\frac{1}{N_{i}^{1-a}}(1-a) C_{i}^{-a}\right)=(1-a) C_{i}^{-a} \frac{1}{2} f B_{i} b \frac{1}{N_{j}^{1-a}}(1-a) C_{j}^{-a} .
\end{gathered}
$$

Then:

$$
\begin{array}{r}
\frac{-\theta^{2} U_{i}}{\theta C_{i}^{2}} \geq \sum_{j \neq i}\left|\frac{\theta^{2} U_{i}}{\theta C_{j} \theta C_{i}}\right| \Leftrightarrow \\
\frac{1}{2}\left[F D_{i} P_{i}+B_{i} b-f \sum_{j \neq i}\left(\frac{C_{j}}{N_{j}}\right)^{1-a} B_{i} b\right] \frac{1}{N_{i}^{1-a}}(1-a) a C_{i}^{-a-1} \\
\geq(1-a) C_{i}^{-a} \frac{1}{2} f B_{i} b \sum_{j \neq i} \frac{1}{N_{j}^{1-a}}(1-a) C_{j}^{-a} \Leftrightarrow \\
{\left[F D_{i} P_{i}+B_{i} b-f \sum_{j \neq i}\left(\frac{C_{j}}{N_{j}}\right)^{1-a} B_{i} b\right] a C_{i}^{-a-1} \geq} \\
C_{i}^{-a} f B_{i} b \sum_{j \neq i} \frac{1}{N_{j}^{1-a}}(1-a) C_{j}^{-a} \Leftrightarrow \frac{a}{C_{i}}\left[F D_{i} P_{i}\right. \\
\left.+B_{i} b-f B_{i} b \sum_{j \neq i}\left(\frac{C_{j}}{N_{j}}\right)^{1-a}\right] \geq(1-a) f B_{i} b \sum_{j \neq i} \frac{1}{N_{j}^{1-a}} C_{j}^{-a} .
\end{array}
$$

Then, we provide two bounds:

$$
\begin{array}{r}
B_{i} b-f B_{i} b \sum_{j \neq i}\left(\frac{C_{j}}{N_{j}}\right)^{1-a} \geq 0, \\
\sum_{j \neq i} \frac{1}{N_{j}^{1-a}} C_{j}^{-a} \leq M-1 .
\end{array}
$$

For the first bound, we use the fact that $f \leq \frac{1}{M-1}$ and $C_{j} \leq N_{j}$. For the second bound, the quantity $\frac{1}{N_{j}^{1-a}} C_{j}^{-a}$ is a decreasing function of $C_{j}$, getting the maximum value at the minimum possible value of $C_{j}$. If $C_{j}$ is at least 1 , then the maximum value of the quantity is $\frac{1}{N_{j}^{1-a}}$, which is maximized when $N_{j}=1$, i.e., when this CP has just one item to sell. Therefore, for the $M-1 \mathrm{CPs}$, the maximum value of is $(M-1) \cdot 1=M-1$.

By using the first bound, we get that:

$\min \left\{\frac{a}{C_{i}}\left[F D_{i} P_{i}+B_{i} b-f B_{i} b \sum_{j \neq i}\left(\frac{C_{j}}{N_{j}}\right)^{1-a}\right]\right\}=\frac{a}{C_{i}} F D_{i} P_{i}$.

By using the second bound, we get that:

$\max \left\{(1-a) f B_{i} b \sum_{j \neq i} \frac{1}{N_{j}^{1-a}} C_{j}^{-a}\right\}=(1-a) \frac{1}{M-1} B_{i} b(M-1)$.

Since $(1) \geq(2)$, we get that:

$$
\begin{gathered}
\frac{a}{C_{i}} F D_{i} P_{i} \geq(1-a) \frac{1}{M-1} B_{i} b(M-1) \Leftrightarrow \\
F \geq \frac{1-a}{a} \frac{C_{i} B_{i} b}{D_{i} P_{i}} \Leftrightarrow F \geq \frac{1-a}{a} \frac{N_{i} B_{i} b}{D_{i} P_{i}},
\end{gathered}
$$

where, in the last step, we also use the fact that the maximum value of $C_{i}$ is equal to $N_{i}$. Since $F$ is a global constant and this condition should hold for each CP $i$, then, we get that:

$$
F \geq \frac{1-a}{a} \max _{i}\left\{\frac{N_{i} B_{i} b}{D_{i} P_{i}}\right\}
$$

It is worth mentioning that in order to compute the maximum value of $F$ there should be some offline exchange of messages among the CPs with the ISP being the one that acts as the mediator. Moreover, though the above condition is necessary and sufficient for the uniqueness of the NE, $F$ should be by definition greater than or equal to $(M-1) f$. Therefore, in case that the lower bound for the existence of the NE is lower than the minimum possible value of $F$, then $F$ can get any value in its domain.

The uniqueness of the NE is of interest since we can predict the outcome of the game. In the next section, we examine how we can obtain this outcome.

\subsubsection{A Best Response Dynamics Scheme}

In this section, we propose a best response dynamics scheme [3], where each CP $i$ updates iteratively his cache size $C_{i}$ aiming at maximizing his utility function $U_{i}$. Though, in general, such a scheme may not converge to a NE, in concave games that admit a unique NE, it is guaranteed that it will converge to the NE. 


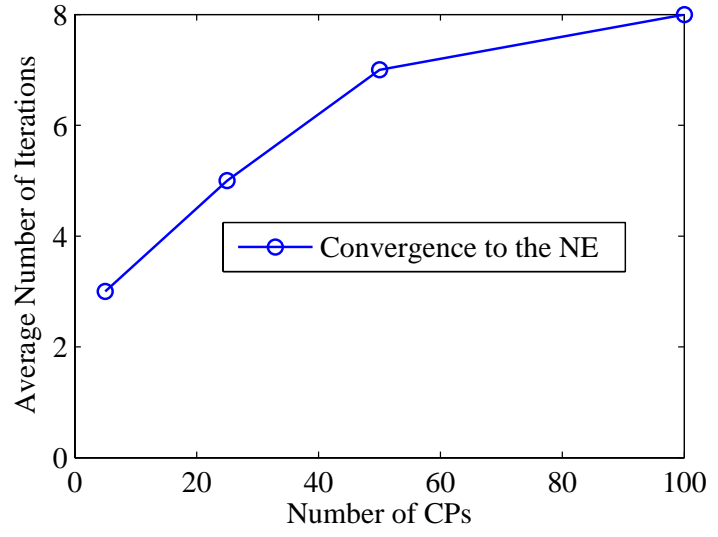

Figure 3: Average number of iterations needed so that the best response dynamics scheme converges to the unique NE.

The cache size at time $t+1$ can be computed using an iterative scheme by taking into consideration the strategies of all other CPs at time $t$ and is given by the following formula:

$$
\begin{array}{r}
C_{i}(t+1)=\max \left\{N_{i}, \sqrt[a]{\frac{(1-a) K_{i}(t)}{s N_{i}^{1-a}}}\right\}, \\
K_{i}(t)=\left(F D_{i} P_{i}+B_{i} b-f \sum_{j \neq i}\left(\frac{C_{j}(t)}{N_{j}}\right)^{1-a} B_{i} b\right) .
\end{array}
$$

This iterative scheme arises by taking the first derivative of the utility function $U_{i}$ and setting it equal to zero in order to compute the local maximum points; in our case, there is a unique maximum point (this was the case with the one $\mathrm{CP}$ as well). As usually, a best response dynamics scheme requires some exchange of messaging among the players to decide upon their new strategies. In our case, each CP $i$ needs to know the sum of the hit rates of all other CPs to update his strategy and the ISP could provide this piece of information; note that the CPs do not need to know the exact hit rate of each CP, they suffice to learn their sum.

We have simulated the best response dynamics scheme to examine the number of iterations needed to converge to the $\mathrm{NE}$ for scenarios that consisting of 5 to $100 \mathrm{CPs}$. For each scenario, we have tuned each parameter that affects the NE convergence starting from very small values and increasing them to very big values and we have taken all possible combinations of them. As shown in Figure 3, the best response dynamics scheme converges fast to the NE, demanding less than 8 iterations on average, even when there are $100 \mathrm{CPs}$.

\section{CONCLUSIONS AND DISCUSSION}

Using fundamental concepts of game theory to analyze networked markets is not only useful from a theoretical perspective but it can also lead to practical contributions in network economics problems. In our work, we analyzed the economics of the widely used idea of caching under the prism of coalitional game theory, examining whether the ISP and the CPs have motivation to share the cache cost/profit. By using standard solution concepts, we showed that a fair and efficient profit sharing can be achieved. On top of the coalitional game, a non-cooperative game among the CPs arises and, through our analysis, we have shown that a unique NE exists and we have proposed a scheme that converges to it in few iterations.

Two promising applications of our work would be in the context of i) Content Delivery Network Providers that store the content of the CPs in order to serve the ISP users and ii) Information-Centric Networks, where content is located by name instead of by location and every node can cache and serve the content [9]. Economic aspects for caching and sharing contents in such networks is an active field of research; indicative results related to our work in such networks with two competing ISPs include revenue-maximizing caching strategies [5] and NE pricing policies [7].

Other directions for future research include: i) the stability analysis of the Shapley value for the case in which there are multiple CPs (e.g., with the view to showing under which conditions this is a convex game [8]), and ii) the study of the complementary problem of the optimal caching policy from the side of the ISP where, based on the different caching policies, network neutrality issues may also appear and should be analyzed.

\section{ACKNOWLEDGEMENT}

This work has been performed in the framework of the H2020 project METIS-II co-funded by the EU.

\section{REFERENCES}

[1] A. Dhamdhere and C. Dovrolis. Twelve years in the evolution of the Internet ecosystem. IEEE/ACM Transactions on Networking, 19(5):1420-1433, 2011.

[2] S.-E. Elayoubi and J. Roberts. Performance and cost effectiveness of caching in mobile access networks. In Proc. 2nd International Conference on Information-Centric Networking, pages 79-88, 2015.

[3] S. Lasaulce, M. Debbah, and E. Altman. Methodologies for analyzing equilibria in wireless games. IEEE Signal Processing Magazine, 26(5):41-52, 2009.

[4] R. T. Ma, D. M. Chiu, J. C. Lui, V. Misra, and D. Rubenstein. On cooperative settlement between content, transit, and eyeball internet service providers. IEEE/ACM Transactions on Networking, 19(3):802-815, 2011.

[5] P. Maillé, G. Simon, and B. Tuffin. Impact of revenue-driven $\mathrm{CDN}$ on the competition among network operators. In Proc. 11th International Conference on Network and Service Management (CNSM), pages 163-167, 2015.

[6] W. B. Norton. The evolution of the US Internet peering ecosystem. Equinix white papers, 2004.

[7] T.-M. Pham, S. Fdida, and P. Antoniadis. Pricing in Information-Centric Network interconnection. In Proc. 12th IFIP Networking Conference, 2013.

[8] W. Saad, Z. Han, M. Debbah, A. Hjorungnes, and T. Basar. Coalitional game theory for communication networks. IEEE Signal Processing Magazine, 26(5):77-97, 2009.

[9] G. Xylomenos, C. N. Ververidis, V. A. Siris, N. Fotiou, C. Tsilopoulos, X. Vasilakos, K. V. Katsaros, and G. C. Polyzos. A survey of information-centric networking research. IEEE Communications Surveys 8 Tutorials, 16(2):1024-1049, 2014. 